\title{
A case of atrial septal defect associated with anomalous sinoatrial node artery in pulmonary atresia with intact ventricular septum
}

\author{
Angela Di Candia ${ }^{1}$, BIAGIO CASTALDI ${ }^{2}$, Domenico Sirico ${ }^{2}$, and Giovanni Di Salvo ${ }^{3}$ \\ ${ }^{1}$ University of Padua \\ ${ }^{2}$ University of Padova Faculty of Medicine and Surgery \\ ${ }^{3}$ Second University of Naples
}

January 28, 2021

\begin{abstract}
An 11-year-old boy affected by pulmonary atresia with intact ventricular septum (AP-IVS) was listed for percutaneous pulmonary valvuloplasty and closure of multi-fenestrated atrial septal defect (ASD). Intraprocedural transesophageal echocardiography arose the suspect of abnormal coronary artery pattern while selective angiography documented a single sinoatrial node artery (SANa) with an unusual retro-aortic course. As consequence, we proceeded to effectively close the defects with a not self-centering device placed in the most central side hole. This case supports the hypothesis that sometimes arrhythmic complication during ASD closure procedures might be due to unrecognized injury of the SANa.
\end{abstract}

A case of atrial septal defect associated with anomalous sinoatrial node artery in pulmonary atresia with intact ventricular septum

Angela Di Candia, $\mathrm{MD}^{1}$, Biagio Castaldi, $\mathrm{MD}, \mathrm{PhD}^{1}$, Domenico Sirico, $\mathrm{MD}^{1}$, Giovanni Di Salvo, MD, PhD, FESC $^{1}$

Pediatric Cardiology Unit, Department of Woman and Child's Health, University of Padua, Italy.

Corresponding author : Biagio Castaldi

Pediatric Cardiology Unit, Department of Woman and Child's Health, University of Padua

Via Giustiniani 2, 35128 Padua, Italy

Fax number: +39 0498218089; Tel: +39 0498213558; email: b.castaldi@yahoo.it

Short title : Anomalous sinoatrial node artery in multifenestrated ASD

Keywords: Atrial septal defect; Sinus node coronary artery; Gore septal device; Congenital heart disease; Interventional cardiology

Disclosure : none

\section{Abstract}

An 11-year-old boy affected by pulmonary atresia with intact ventricular septum (AP-IVS) was listed for percutaneous pulmonary valvuloplasty and closure of multi-fenestrated atrial septal defect (ASD). Intraprocedural transesophageal echocardiography arose the suspect of abnormal coronary artery pattern while selective angiography documented a single sinoatrial node artery (SANa) with an unusual retro-aortic course. As consequence, we proceeded to effectively close the defects with a not self-centering device placed in the 
most central side hole. This case supports the hypothesis that sometimes arrhythmic complication during ASD closure procedures might be due to unrecognized injury of the SANa.

\section{Text}

The sinoatrial node artery (SANa) has significant anatomic variability and some variants have been described to be associated with a greater risk of iatrogenic injury during surgical or percutaneous procedures.

An 11-year-old boy affected by pulmonary atresia with intact ventricular septum was evaluated at our center for a follow-up visit. He had previously undergone multiple percutaneous pulmonary valvuloplasty. Given the echocardiographic finding of significant gradient through the pulmonary valve and spontaneous right-toleft atrial shunt, the boy was listed for the fourth percutaneous pulmonary valvuloplasty and percutaneous closure of multi-fenestrated atrial septal defect (ASD). The largest ASD was located antero-superiorly just behind the Aorta. A static sizing by using an $18 \mathrm{~mm}$ Amplatzer@ Sizing Balloon (Abbott, Plymouth, MN) showed a stretched diameter of $11 \mathrm{~mm}$. In addition, a patent foramen ovale (PFO) and a small, more central, ASD were found. Intraprocedural transesophageal echocardiography (TEE) showed an unusual flow behind the non-coronary aortic sinus, rising the suspect of abnormal coronary artery pattern (figure 1). For this reason, selective coronary angiography was performed. Left coronary angiogram documented a single SANa having a course compatible with the echocardiographic finding. Indeed, this artery appeared to originate from the left circumflex coronary artery (LCCA), turn posteriorly, run close the non-coronary aortic cusp, cross the antero-superior interatrial groove and continue with a precaval course towards the sinoatrial node (figure 2). Coronary angiography highlighted the proximity relationship between the ASD and the SANa, the latter intersecting the antero-superior rim of the interatrial septum. On the basis of the haemodynamic data collected, after successful pulmonary valvuloplasty, we proceeded with the ASD closure by using a 30 mm Gore @ Septal Occluder device (WL Gore \& Associates, Flagstaff, AZ) deployed in the central side hole rather than a self-centering device in the larger antero-superior ASD.

At the end of the procedure, good conformation of the device and absence of residual shunts were documented. The left coronary artery (LCA) was rechecked by selective coronary angiography: the SANa ran adjacent to the device, without being touched by it (figure 3). The ECG didn't show any changes in the heart rhythm. The follow-up was uneventful.

Pulmonary atresia with intact ventricular septum (PA-IVS) is a rare congenital heart disease (3\% of congenital heart disease, with an incidence of $4-8$ out of 100,000 live births). It can be associated with anomalies in the development of the coronary arteries, linked to the peculiar pathophysiology that leads to the creation of ventriculo-coronary fistulas or atretic coronary tracts. ${ }^{1}$ To our knowledge, no anomalies in the course of the SANa associated with PA-IVS have been reported to date. ${ }^{2}$

Autoptic or imaging study described a wide anatomical variability of the SANa in the general population, regarding either the origin and the course. ${ }^{3-6}$ Most commonly, SANa arises from right coronary artery (RCA) (54-68\% of cases) or from LCCA (22-40\% of cases). Regarding the relationship with the superior vena cava, SANa can present a retrocaval or a precaval or a pericaval course. The former is described to be about $47 \%$ of total cases and is the most frequent in the case of origin from the LCA/LCCA in the European population. The precaval and pericaval courses follow, representing respectively $39 \%$ and $14 \%$ of the total cases. High risk anatomical variants, such as S-shaped artery, are described to be more associated with possible sinus node dysfunction during surgical or percutaneous procedures, which involve a manipulation of atrial walls or interatrial septum. ${ }^{3,4}$ They must be taken into account to prevent iatrogenic injuries. In fact, the damage of the SANa is associated with dysfunction of the sinus node and the onset of arrhythmias, such as junctional rhythm or supraventricular tachycardia. ${ }^{7-9}$

Saremi et al (2008) in a study of 250 patients undergoing coronary CT, described $13.9 \%$ of cases having the S-shaped variant, which originated from LCCA, turned posteriorly and passed in a groove at the junction between the orifice of the left upper pulmonary vein and the mouth of the left atrial appendage. Only one third of these cases presented a precaval course. ${ }^{4}$ 
In our case, conversely, the SANa didn't embrace the left atrial appendage and the superior left pulmonary vein: after detaching from the LCCA, our SANa immediately turned posteriorly with an acute angle towards the non-coronary aortic cusp, crossed the antero-superior rim of the interatrial septum and continued with a precaval course towards the atrial sinus node (figure 4).

In conclusion, our case describes a new variant of SANa course, which crossed the antero-superior groove of the atrial septum, found in a patient with AP-IVS. Despite a coronary angiography is not routinely performed before ASD closure, a careful echocardiographic assessment of the defect, and the surrounding structure as well, is mandatory to minimize the risk of procedural complications. Indeed, it can be speculated that at least part of the ASD closure procedures complicated by rhythm alterations $(0,6-2,7 \%$ of cases $),{ }^{10}$ such as junctional rhythm or supraventricular tachycardias, might sometimes be due to an unrecognized SANa course anomalies.

\section{References}

1. Chikkabyrappa SM, Loomba RS, Tretter JT. Pulmonary Atresia With an Intact Ventricular Septum: Preoperative Physiology, Imaging, and Management. Semin Cardiothorac Vasc Anesth. 2018;22(3):245-255. doi:10.1177/1089253218756757.

2. Daubeney PEF, Delany DJ, Anderson RH, et al. Pulmonary atresia with intact ventricular septum. Journal of the American College of Cardiology. 2002;39(10):1670-1679. doi:10.1016/S0735-1097(02)01832-6.

3. Berdajs D, Patonay L, Turina MI. The clinical anatomy of the sinus node artery. The Annals of Thoracic Surgery. 2003;76(3):732-735. doi:10.1016/S0003-4975(03)00660-X.

4. Saremi F, Channual S, Abolhoda A, Gurudevan SV, Narula J, Milliken JC. MDCT of the S- Shaped Sinoatrial Node Artery. American Journal of Roentgenology. 2008;190(6):1569- 1575. doi:10.2214/AJR.07.3127.

5. Vikse J, Henry BM, Roy J, et al. Anatomical Variations in the Sinoatrial Nodal Artery: A Meta-Analysis and Clinical Considerations. Cappello F, ed. PLoS ONE. 2016;11(2):e0148331. doi:10.1371/journal.pone.0148331.

6. Vikse J, Henry BM, Roy J, et al. Correction: Anatomical Variations in the Sinoatrial Nodal Artery: A Meta-Analysis and Clinical Considerations. PLoS ONE. 2016;11(3):e0150051. doi:10.1371/journal.pone.0150051.

7. Hardie EL, Jones SB, Euler DE, Fishman DL, Randall WC. Sinoatrial node artery distribution and its relation to hierarchy of cardiac automaticity. American Journal of Physiology-Heart and Circulatory Physiology. 1981;241(1):H45-H53. doi:10.1152/ajpheart.1981.241.1.H45.

8. de Groot NMS. The relationship between sinus node dysfunction, bradycardia-mediated atrial remodelling, and post-operative atrial flutter in patients with congenital heart defects. European Heart Journal. 2006;27(17):2036-2037. doi:10.1093/eurheartj/ehl150.

9. Ando' G. Acute thrombosis of the sinus node artery: arrhythmological implications. Heart. 2003;89(2):5e55. doi:10.1136/heart.89.2.e5.

10. Jalal Z, Hascoet S, Gronier C, et al. Long-Term Outcomes After Percutaneous Closure of Ostium Secundum Atrial Septal Defect in the Young. JACC: Cardiovascular Interventions. 2018;11(8):795-804. doi:10.1016/j.jcin.2018.01.262.

\section{Figure legend}

Figure 1: Aortic short axis transesophageal echocardiography showing main atrial septal defect (hashtag) and an unusual flow behind the non-coronary aortic sinus (asterisk).

Figure 2 : Antero-posterior (a) and lateral (b) views of selective left coronary angiography. Sinoatrial node artery is marked by asterisk. 
Figure 3 : Antero-posterior (a) and lateral (b) views of selective left coronary angiography after Gore(r) Septal Occluder device deployment. Sinoatrial node artery is marked by asterisk.

Figure 4 : Example drawing of the sinoatrial node artery (in red) described in the case report. SAN: sinoatrial node; SVC: superior cava vein; PPVV: pulmonary veins; SANa: sinoatrial node artery; LCCA: left circumflex coronary artery; LAD: left anterior descending coronary artery; LMCA: left main coronary artery; PV: pulmonary valve; AV: aortic valve.

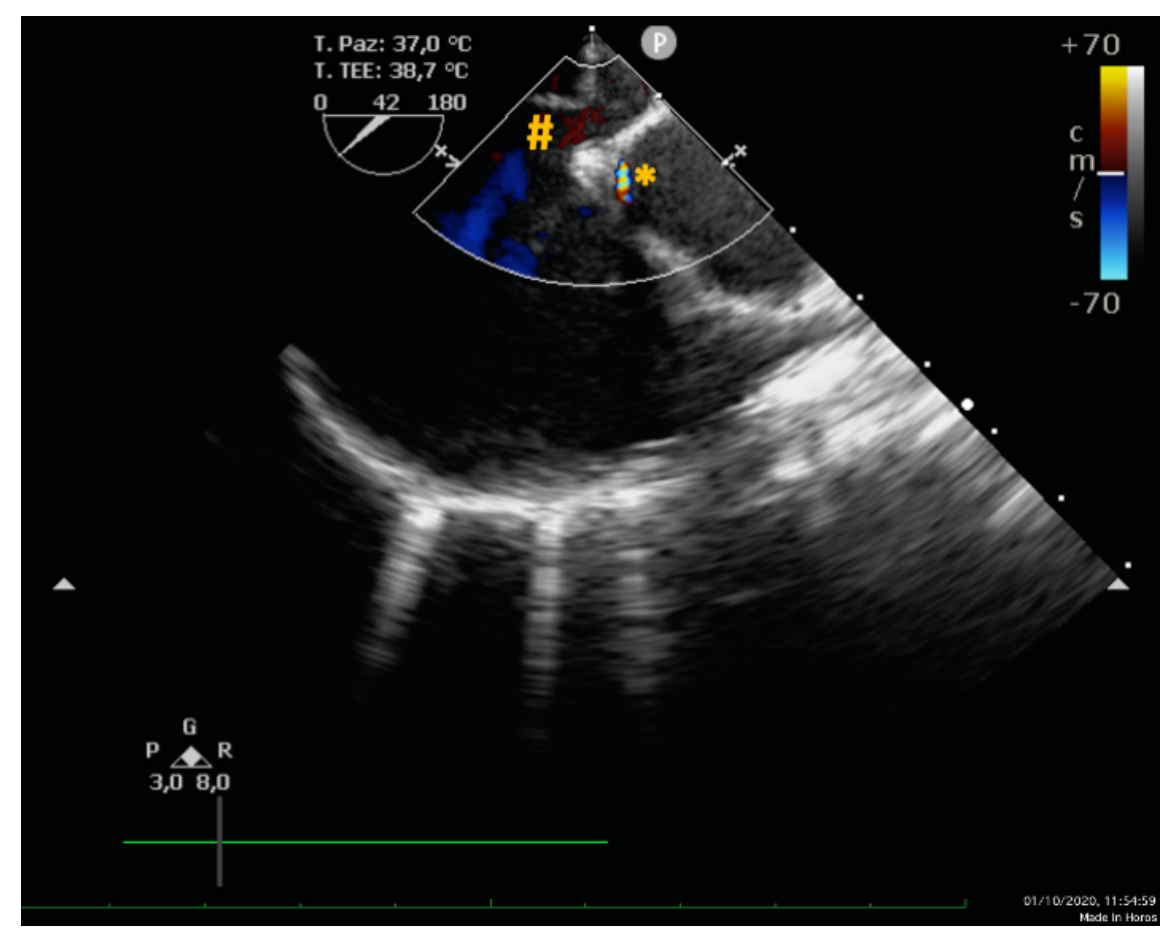



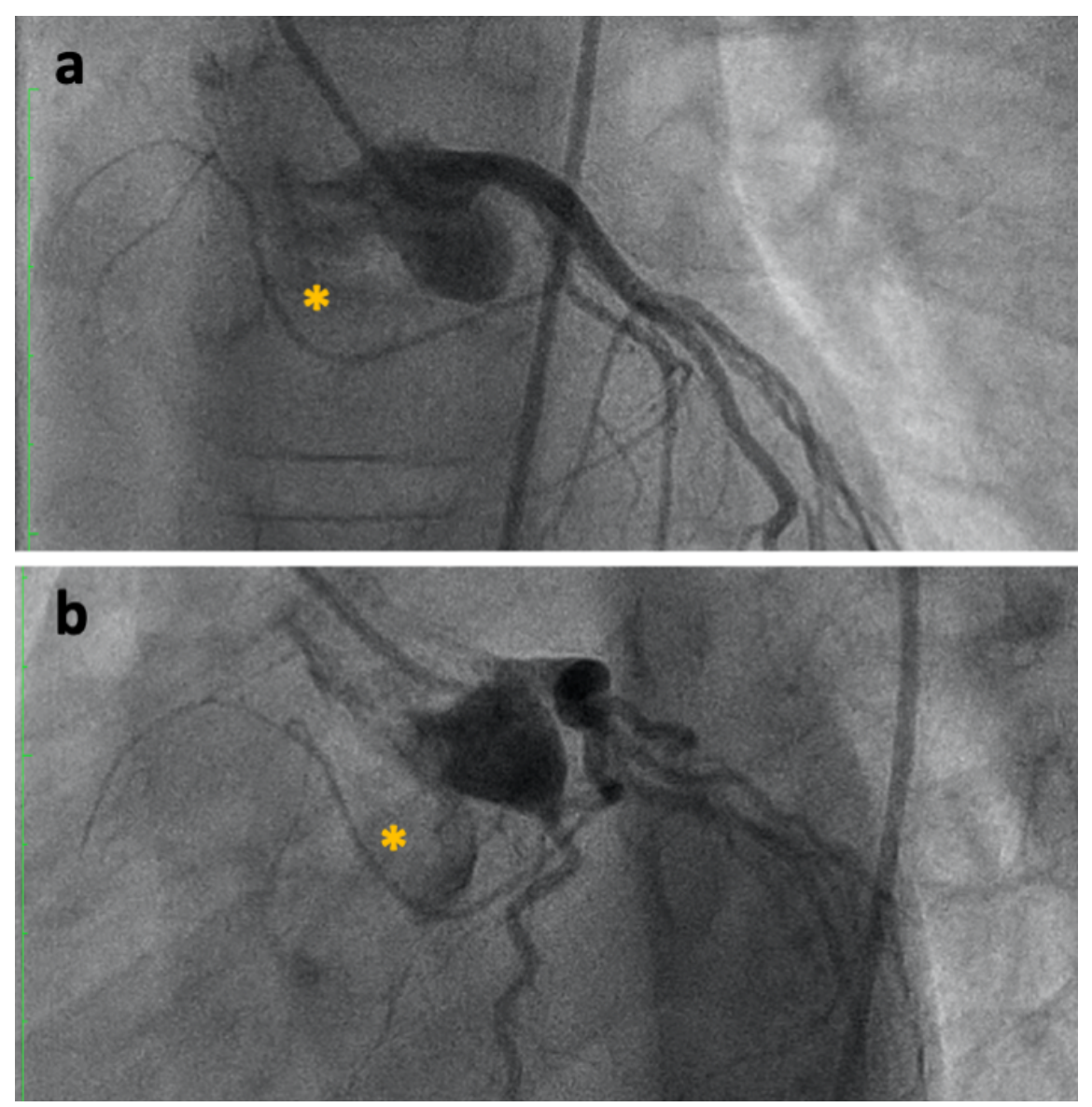

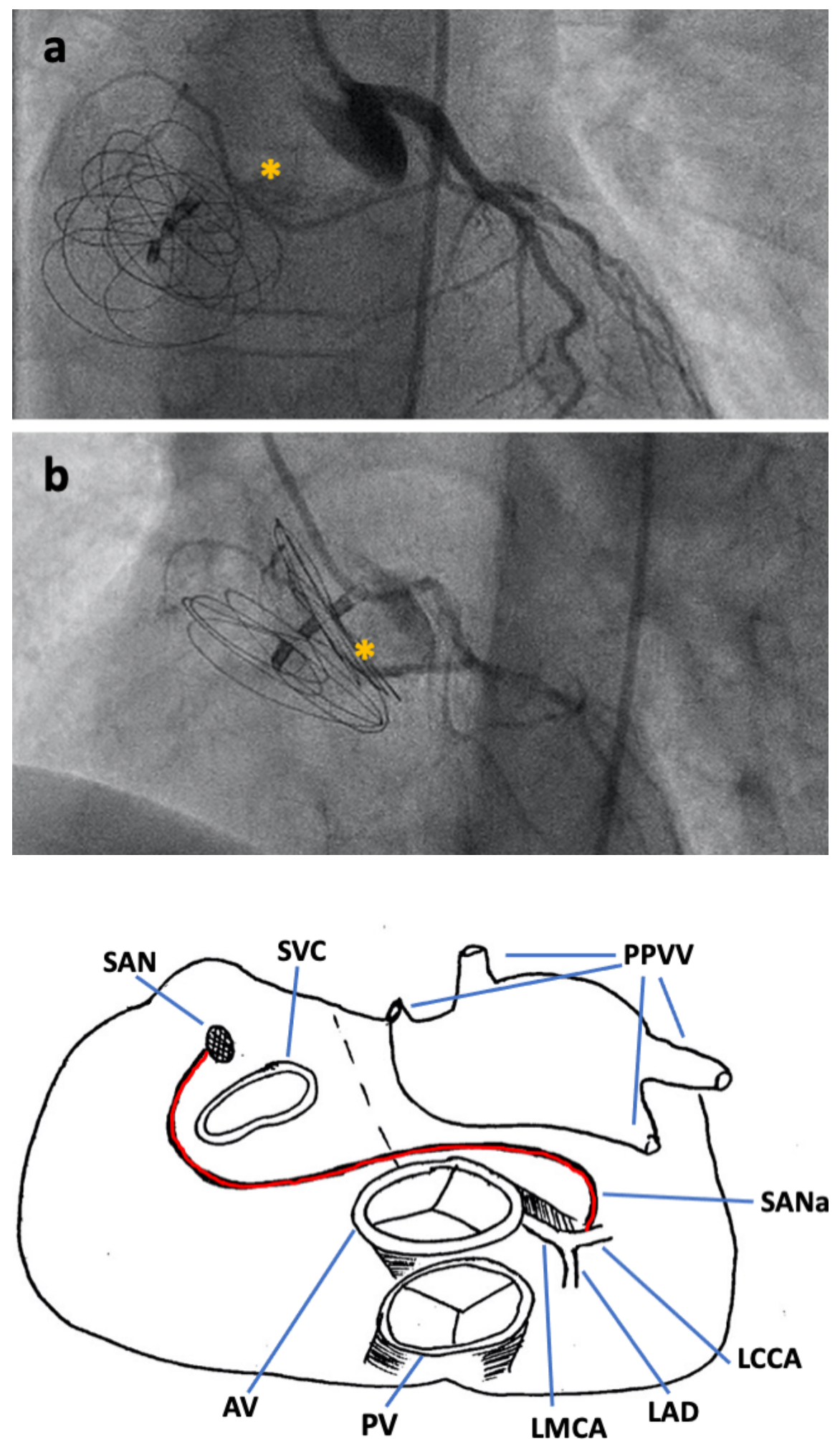